Saeid Eslami

Zhila Taherzadeh

Marcus J. Schultz

Ameen Abu-Hanna

\section{Glucose variability measures and their effect on mortality: a systematic review}

Received: 7 September 2010

Accepted: 28 December 2010

Published online: 29 January 2011

(C) The Author(s) 2011. This article is published with open access at Springerlink.com

\section{S. Eslami (®) - A. Abu-Hanna} Department of Medical Informatics, Academic Medical Center, University of Amsterdam, Meibergdreef 15, 1105 AZ Amsterdam, The Netherlands e-mail: s.eslami@amc.uva.nl

\section{Z. Taherzadeh}

Department of Internal Medicine, Academic Medical Center, University of Amsterdam, Amsterdam, The Netherlands

\section{J. Schultz}

Department of Intensive Care, Academic Medical Center, University of Amsterdam, Amsterdam, The Netherlands

M. J. Schultz

Laboratory of Experimental Intensive Care and Anesthesiology, Academic Medical

Center, University of Amsterdam,

Amsterdam, The Netherlands
M. J. Schultz HERMES Critical Care Group, Amsterdam, The Netherlands
Abstract Objective: To systemat- ically review the medical literature on the association between glucose var- iability measures and mortality in critically ill patients. Meth-
ods: Studies assessing the association between a measure of glucose variability and mortality that reported original data from a clinical trial or observational study on criti- cally ill adult patients were searched in Ovid MEDLINE ${ }^{\circledR}$ and Ovid EM- BASE $^{\circledR}$. Data on patient populations, study designs, glucose regulations, statistical approaches, outcome mea- sures, and glucose variability indicators (their definition and appli- cability) were extracted.
Result: Twelve studies met the inclusion criteria; 13 different indi- cators were used to measure glucose variability. Standard deviation and the presence of both hypo- and hyperglycemia were the most com- mon indicators. All studies reported a statistically significant association

between mortality and at least one glucose variability indicator. In four studies both blood glucose levels and severity of illness were considered as confounders, but only one of them checked model assumptions to assert inference validity. Conclu-

sions: Glucose variability has been quantified in many different ways, and in each study at least one of them appeared to be associated with mortality. Because of methodological limitations and the possibility of reporting bias, it is still unsettled whether and in which quantification this association is independent of other confounders. Future research will benefit from using an indicator reference subset for glucose variability, metrics that are linked more directly to negative physiological effects, more methodological rigor, and/or better reporting.

Keywords Blood glucose . Blood glucose variability · Glycemic control · Mortality . Systematic review

\section{Introduction}

Glucose control aiming at normoglycemia, i.e., blood glucose levels (BGLs) of $80-110 \mathrm{mg} / \mathrm{dl}$, which is frequently referred to as "tight glycemic control" (TGC), decreased morbidity and mortality of critically ill patients
[1]. Later studies investigated the effect of various forms of glucose control on mortality and morbidity and reported that no beneficial effects of these interventions were found [2-5]. Some studies showed that their implementation of glucose control with insulin comes with the risk of hypoglycemia $[6,7]$, others did not $[8,9]$. 
Partly in order to reconcile the findings of these seemingly contradictory results, confounders-correlating with both blood glucose levels and mortality-and other possible factors associated with mortality were sought. One of these potential confounders or possible factors associated with mortality is blood glucose variability, which in essence quantifies the spread in a set of blood glucose levels.

The underlying idea for considering glucose variability is that it, similar to hyperglycemia, can be associated with mortality by increasing oxidative stress, neuronal damage, mitochondrial damage, and coagulation activity [10-13]. It has been shown that rapid fluctuations of BGLs increase oxidative stress and are more detrimental than sustained hyperglycemia. In consequence, investigating the role of blood glucose variability as a new target in blood glucose control was suggested [13, 14]. However, one may distinguish between various sources of variability in BGLs. The variability can be relatively controllable, induced by intervention, or its can effectively appear random (e.g., due to patient condition changes). All variability that exposes the patient to negative physiological consequences is to be avoided.

The main objective of this systematic review was to identify and summarize published studies on glucose variability and their association with mortality in critically ill patients. This summary includes an overview of all the glucose variability indicators used along with a methodological examination of the studies. This review may form a basis for future developments of a standard list of well-defined glucose variability indicators and methodological considerations thereby contributing to the comparability and quality of new studies.

\section{Materials and methods}

We searched for relevant English-language articles based on keywords in title, abstract, and MeSH terms, using Ovid MEDLINE $^{\circledR}$, Ovid MEDLINE ${ }^{\circledR}$ In-Process (1950 to April 30, 2010) and Ovid EMBASE ${ }^{\circledR}$ (1980 to April 30, 2010). The final literature search was performed on May $10,2010$.

Figure 1 shows the search strategy. Searching was supplemented by scanning bibliographies from identified articles. Two authors independently examined all titles and abstracts. Discrepancies among the two authors were resolved by consensus involving a third author. Articles were included if they reported original data from a clinical trial or observational study on critically ill patients and concerned the association between glucose variability and mortality. Opinion papers, surveys, and letters were excluded.

From the selected papers, the same two authors extracted data on the patient population, study design, glucose regulation, statistical approach, outcome measures, and glucose variability indicators (their definition and applicability). Discrepancies between the two authors were again resolved by consensus after involving the

Fig. 1 The search flow diagram

glucose AND (variability OR variation) AND (critical illness OR critical care OR intensive care)

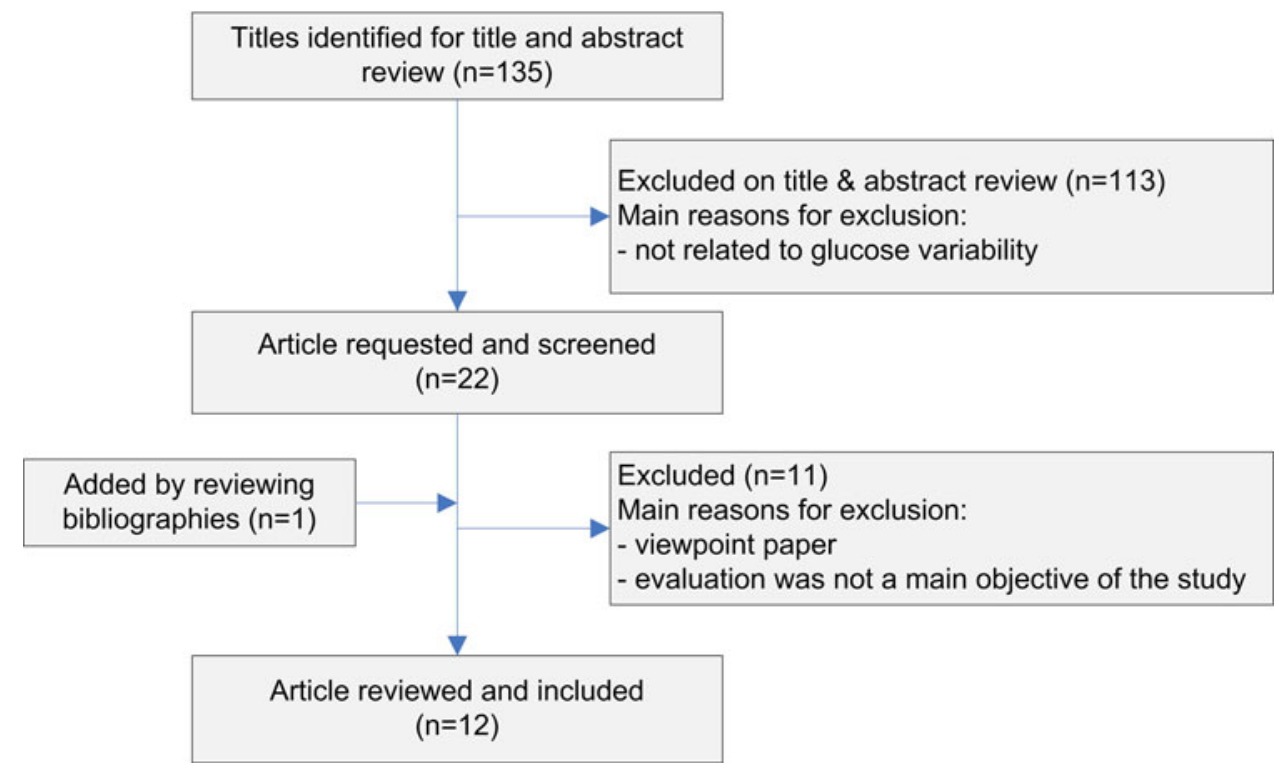


same third author. Heterogeneity of the retrieved studies in terms of the extracted data (patient populations, study designs, variability indicators etc.) was informally assessed to determine whether a meta-analysis was justified.

\section{Results}

Searching the online databases resulted in 135 articles. Initial screening of titles and abstracts rendered 22 articles eligible for further full-text review. Inspecting the bibliographies of these articles yielded an extra article. On the basis of the full-text review, 11 studies, including the one added by the bibliographic search, were excluded, leaving 12 articles for detailed analysis. Table 1 uniformly describes these studies.

Table 2 lists and describes the 13 glucose variability indicators used. The most frequently used indicator was the standard deviation (SD) of BGLs per patient, which was employed in 6 out of 12 articles. Five out of six studies reported on a statistically significant positive association between SD and mortality. The populations in these studies were sepsis patients (2/6) and mixed surgical and medical patients (4/6).

The second commonly used indicator is what we call the "hyper-hypo" indicator which is a binary (true or false) variable defined as true for a patient having both hyperglycemia and hypoglycemia measurements during some predefined interval. Two interval-variants of the hyper-hypo indicator were encountered in 3/12 studies: one variant was defined for the whole intensive care unit (ICU) admission and the other for the first $24 \mathrm{~h}$ after admission. Two of the three studies using the hyper-hypo indicator showed a statistically significant association with mortality and the third study reported consistent but non-significant association. However, six different thresholds were used in these three studies for defining hyperglycemia $(>150,>180$, and $>216 \mathrm{mg} / \mathrm{dl})$ and hypoglycemia $(<60,<72$, and $<81 \mathrm{mg} / \mathrm{dl})$. The study populations were also different in these studies and included patients with brain injury, mixed medical/surgical patients, and non-diabetic patients receiving insulin during their admission.

Each of the rest of the eleven indicators was used in only one study. All of them were found to be statistically significantly associated with ICU and/or hospital mortality.

In the majority of studies the association between mortality and the used variability indicator was adjusted for by using linear logistic regression analysis (Table 1). However, different sets of possible confounders were used. Six out of 12 studies used at least one severity of illness indicator as a confounder in the final regression model. Only four studies included the mean or median
BGL as a confounder in the final regression model. In four studies both blood glucose levels and severity of illness were considered as confounders. All studies assumed a linear relationship between (log odds of) mortality and either the blood glucose variability indicator itself or the confounders used, but only one study reported on testing this assumption [15]. A more recent study [16] showed that observed mortality reduction with intensive insulin therapy (IIT) in the original Leuven trials could not be attributed to blood glucose variation defined by SD and mean daily $\delta$ blood glucose. However that study also showed that increased blood glucose variation was associated with mortality irrespective of blood glucose level. Protocol implementation (with or without IIT) and level of adherence to it either were not mentioned or differed from one study to another. In addition, different BGL target ranges were used in the studies that reported on adherence.

\section{Discussion}

The apparent heterogeneity of the retrieved studies does not justify conducting a meta-analysis. Although all 12 studies reported a significant association between mortality and one or more of a set of 13 glucose variability indicators, the study heterogeneity, the methodological limitations, and the possibility of reporting bias do not support an unequivocal evidence for the independence of this association of other factors, let alone its causal nature. To our knowledge, this is the first review of the literature on the association between blood glucose variability and mortality. Because of the current attention to blood glucose variability in critically ill patients, we believe the review to be timely, enabling the dissemination of the lessons learned from the reviewed studies. A limitation of our search is that we only addressed studies that had a main objective of evaluating the association between mortality and glucose variability; we might have missed some studies with a limited focus.

Aside from differences in patient case-mix and in the associated therapy, the diversity of the variability indicators used, and the fact that only few if at all were used together, hampers comparability of the studies and hence of the consolidation of evidence. In addition, and especially because none of the studies had an randomized control trial (RCT) design, one cannot a priori exclude the possibility of a reporting bias, in which only indicators showing associations in a study were reported.

BGL distributions are not necessarily normally distributed nor even symmetric about the mean, especially as the physiologically reported variations are also not necessarily normally distributed. Yet, many studies report variability in terms of, e.g., standard deviation. Inference 


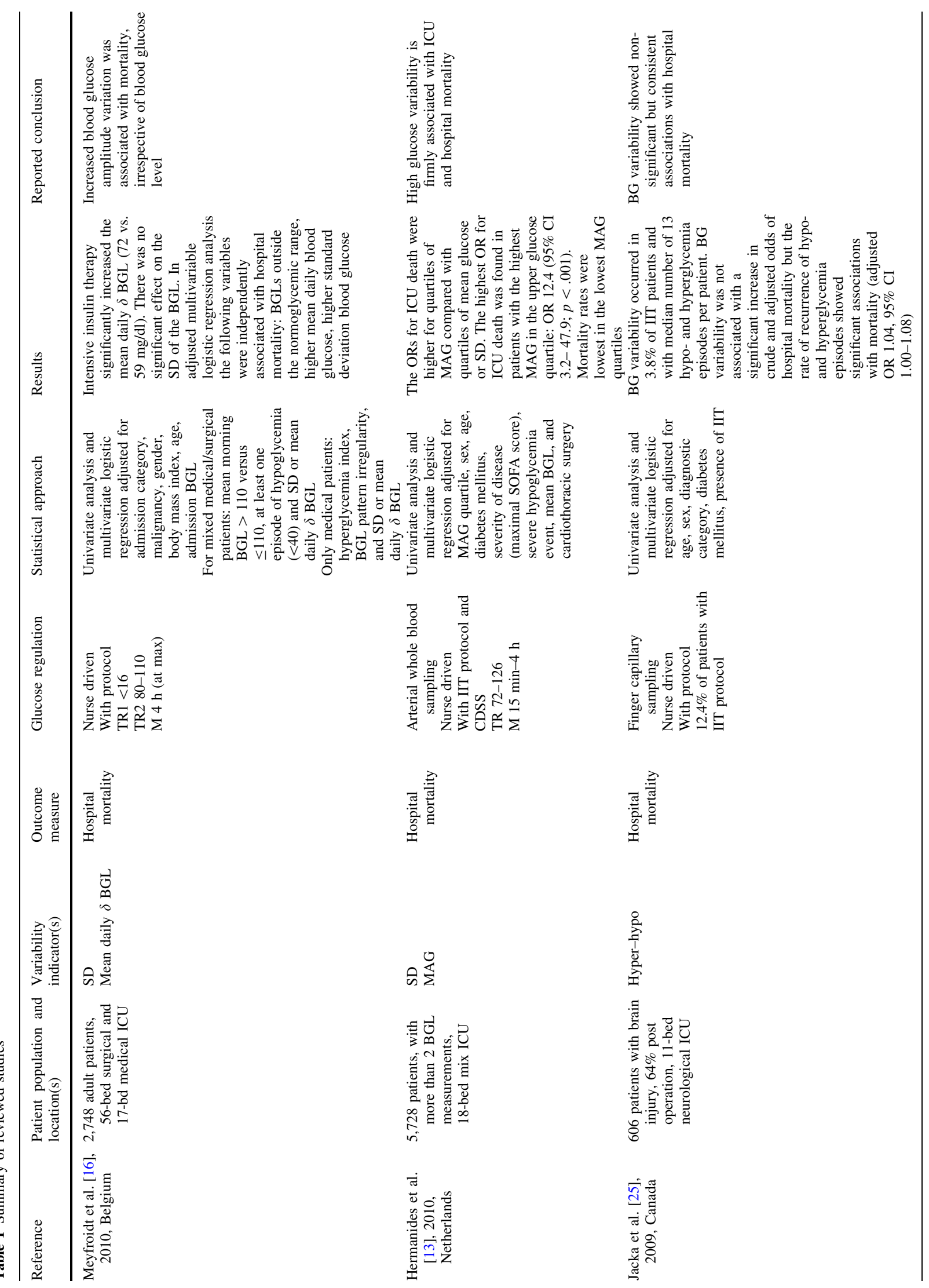




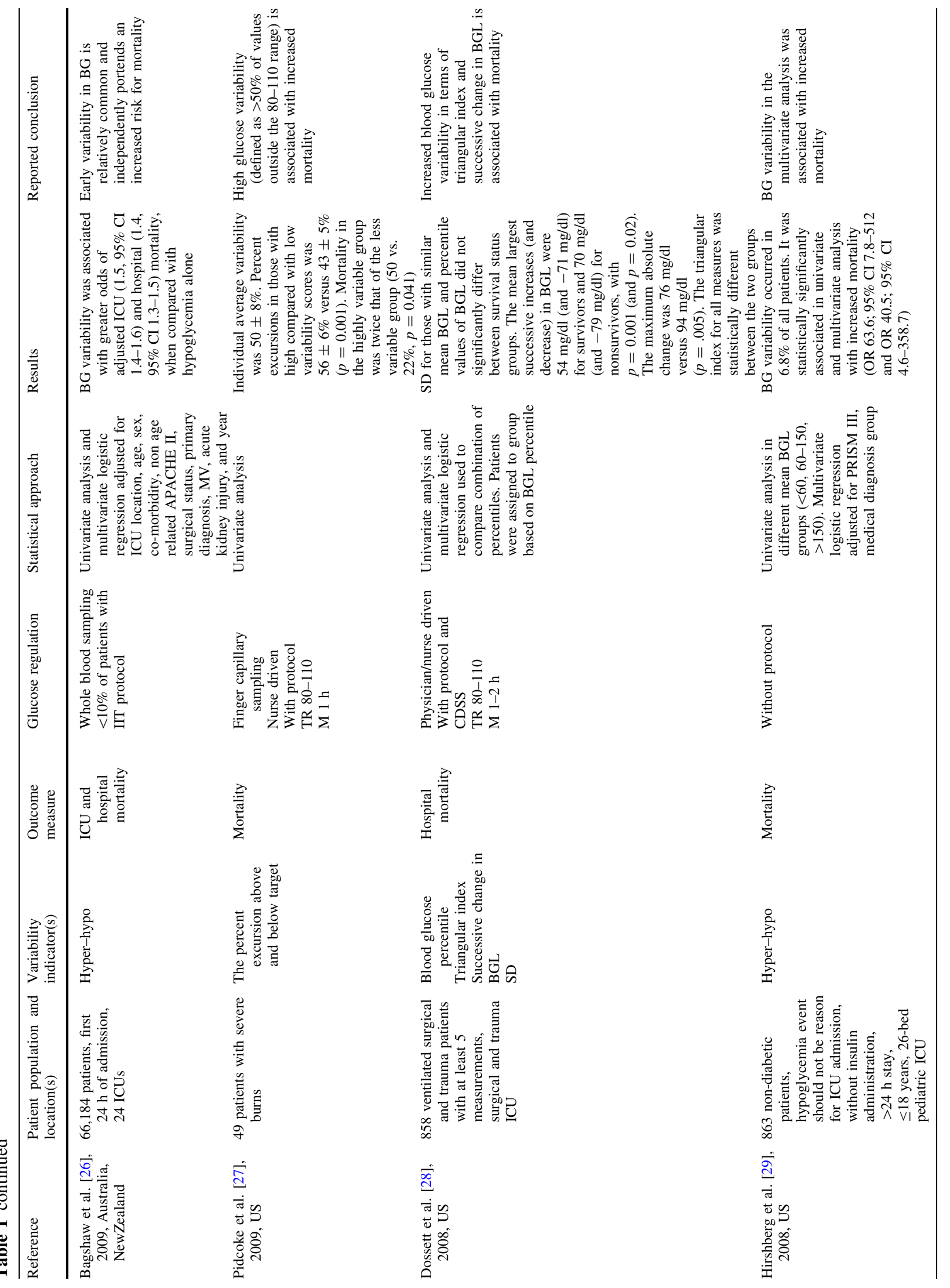









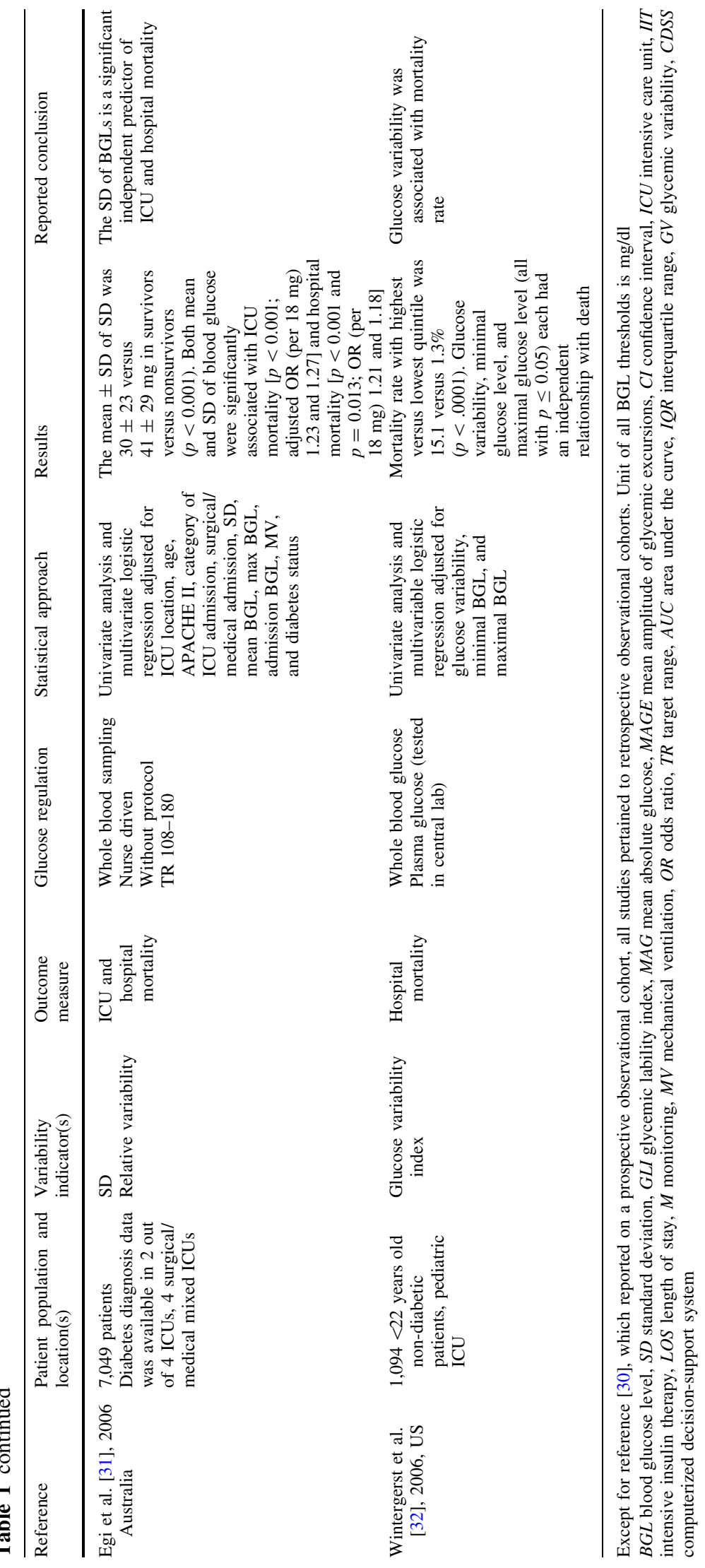


Table 2 List of applied blood glucose variability indicators

\begin{tabular}{|c|c|c|}
\hline Variability indicators & Definition & Ref. \\
\hline Standard deviation & $\begin{array}{l}\text { SD of BGL measurement for each patient during the complete stay in } \\
\text { ICU or during the first } 24 \mathrm{~h} \text { after admission [31] }\end{array}$ & {$[13,15,16,19,30,31]$} \\
\hline Both hypo and hyper & $\begin{array}{l}\text { Defined for a patient as any patient as the presence of both } \\
\text { hyperglycemia }(>150[29],>180[25], \text { or }>216 \mathrm{mg} / \mathrm{dl}[26]) \text { and } \\
\text { hypoglycemia }(<60[29],<72[25], \text { or }<81 \mathrm{mg} / \mathrm{dl}[26]) \text { during the } \\
\text { complete stay in ICU [25], [29], or during the first } 24 \mathrm{~h} \text { after ICU } \\
\text { admission [26] }\end{array}$ & $[25,26], 29]$ \\
\hline Blood glucose percentile & Individual variable ranked, with various percentiles (P50, P95, etc.) & {$[28]$} \\
\hline Glucose variability index & $\begin{array}{l}\text { Mean of absolute difference of sequential BGLs divided by the } \\
\text { difference in BGL collection time }\end{array}$ & [32] \\
\hline GLI & $\begin{array}{l}\text { Squared difference between consecutive BGLs per unit of actual time } \\
\text { between those samples }\end{array}$ & [15] \\
\hline MAG & Mean absolute glucose change per patient per hour & [13] \\
\hline MAGE & $\begin{array}{l}\text { Mean of absolute values of any delta BGL (consecutive values) that are } \\
>1 \text { SD of the entire set of BGLs }\end{array}$ & {$[15]$} \\
\hline Mean daily $\delta$ BGL & Mean of daily difference between minimum and maximum BGL & [16] \\
\hline Relative variability & Coefficient of variability $\left(\mathrm{Glu}_{\mathrm{CV}}=\mathrm{Glu}_{\mathrm{SD}} \times 100 / \mathrm{Glu}_{\mathrm{Ave}}\right)$ & [31] \\
\hline Successive change & $\begin{array}{l}\text { Successive change in BGL calculated by determining the difference in } \\
\text { two consecutive BGLs-the largest successive increase, decrease, and } \\
\text { absolute value were calculated for each patient (regardless of interval } \\
\text { between measures) }\end{array}$ & [28] \\
\hline $\begin{array}{l}\text { Percent excursion above } \\
\text { and below target }\end{array}$ & $\begin{array}{l}\text { Percent excursion (as fraction of the whole) above and below BGL target } \\
\text { with the total number of measurements as the denominator }\end{array}$ & {$[27]$} \\
\hline Triangular index & $\begin{array}{l}\text { Calculated by dividing the maximum sample density distribution of each } \\
\text { histogram for BGL (i.e., the mode) from an individual patient (on a } \\
\text { discrete scale with bin of } 1 \mathrm{mg} / \mathrm{dl} \text { ) by the total number of each } \\
\text { measurement }\end{array}$ & {$[28]$} \\
\hline
\end{tabular}

$B G L$ blood glucose level, $G L I$ glycemic lability index, $M A G$ mean absolute glucose, $M A G E$ mean amplitude of glycemic excursions

based on normality assumptions may then be flawed as they, e.g., would imply the existence of negative BGLs below a number of standard deviations from the mean. The variability itself in exposure to hyper- or hypoglycemia may as well not be symmetric about a mean value. Application of normal statistics to lognormal or skewed distributions can hence yield misleading results.

There should be an agreement on which subset of indicators should be used and reported in future studies but this requires a better understanding of what the indicators intend to measure. This is particularly relevant because the act of measuring BGL is primarily directed to inform future BGL regulation decisions, so BGL measurements would tend to cluster around changes. Some changes are beneficial because they steer BGL towards a target value, others are not. If indicators do not take the direction of a change into account, as is the case, e.g., for the mean absolute glucose (MAG), mean amplitude of glycemic excursions (MAGE), and glycemic lability index (GLI), then they are likely to be sensitive to the sampling frequency of the blood glucose regulation policy and there is a possibility that they, indirectly, also reflect severity of illness. MAG, MAGE, and GLI take order of measurements into account but only MAG and GLI consider the time that elapsed between measurements. SD, hypoglycemia, hyperglycemia, mean daily $\delta$ BGL, and successive change do not take order and timing of measurements into account. Both hypo- and hyperglycemia also depend on the defined threshold. It is unclear which characteristics are responsible for picking up on the variability with the true deleterious biological effect and which are not. To the best of our knowledge there is no study that measured correlation among measures of variability. This merits future research and it can shed light on what is missed when using one measure rather than another.

Recently we suggested that the association of measures such as MAG with mortality may well be blurred by the frequency of observations, and in turn by whether the patients belong to a TGC cohort or not [17]. Three studies $[16,18,19]$ investigated the association between mortality and variability in a TGC and/or a control group as well as in a pooled cohort. In contrast to the Leuven study [16], the SPRINT study [18] showed no significant association between variability and mortality in the SPRINT or TGC arm. Krinsley et al. [19] showed that treatment in the TGC era was negatively correlated to SD. TGC might in this sense be perceived as statistically "decoupling" metrics of variability from mortality within a TGC cohort. Further similar analyses are recommended. In addition, one should realize that there are two statistical ways to view association: testing and estimation. Testing pretends that the answer to the association question is yes or no. In contrast, 
in estimation one simply attempts to quantify the strength of the association, however weak it may be. "Proving" the absence of any association is very hard, but it is easy to quantify its strength. There is a need for new statistical approaches, such as the Bayesian approach, that do not rely on $p$ values and testing, and this facilitates more reliable meta-analysis.

A recent study [20] performed complexity analysis in glycemia time series and showed that loss of complexity, evaluated by detrended fluctuation analysis, is associated with higher mortality. Lack of complexity may signify failure of regulatory systems or extreme consequences of pathophysiological processes. It might be useful to perform complexity analysis, in addition to the traditional variability analysis, in future studies.

Although the majority of studies used linear logistic regression to adjust for confounders, different sets of candidate confounders were used in the studies, again hampering comparability. The choice of these candidates should be motivated by the possible explanations of the suspected association between glucose variability and mortality. Egi et al. [12] state that there are at least three possible explanations for this association. First, less glucose variability could result from more attention in medical and nursing care. Second, less glucose variability may be associated with less severity of illness. Third, glycemic variability might have a true deleterious biological effect in critically ill patients. Hence it is important to always control for the measurement rate per patient (influenced by the blood glucose control protocol and the severity of illness of the patient) as well as severity of illness itself. Otherwise the true deleterious biological effect will be blurred. It is hence beneficial to report on the relationship between a variability indicator and factors that may influence it including measurement rate (or mean interval between measurements) and severity of illness. This was not attempted in the studies. Aside from adjustment in the model, the assumption of linearity between (log odds of) mortality and the indicator and/or the confounders used was not tested when the variables were coded as continuous. By making sure that the assumptions hold, one gets unbiased odds ratio estimates of the variables used.

The course taken in research pertaining to the association between blood glucose variability and mortality is reminiscent of the course taken in researching the association between blood glucose level indicators and the quality of the glucose regulation process. In two recent systematic reviews we showed that many different and even ambiguous quality indicators were used to measure the quality of glucose regulation in these studies which hinders synthesizing evidence [8, 21, 22]. If blood glucose variability is to form a new target in blood glucose control, we need to acknowledge that the term covers various types of variability and define at this stage a uniform set of quality indicators for it that can be used consistently and compared in each study to avoid reporting bias. Second, if some indicators are unambiguously shown to be associated with mortality then we need to develop an approach for influencing them. However this is not trivial especially because other factors, such as mean blood glucose level, should be reckoned with. These important issues should be addressed before conducting new clinical trials on blood glucose variability.

Finally, glycemic metrics in TGC are typically only linked to mortality through a host of other therapies and factors (such as patient condition and age). Linking mortality and glycemia may, however, simply be a "bridge too far" as there is no direct physiological link between glycemia and mortality, except through a range of intermediate physiological processes, which may or may not tip the balance. What seems useful is to have a metric of variability (or level) that links between glycemic control and a clinical outcome that is "closer" to the physiological effects of glycemia than mortality. This link has appeared in some studies [23, 24]. Seeking metrics that are linked more directly to negative physiological effects merits further research.

\section{Conclusion}

The evidence about the independent association between BGL variability and mortality is still unsettled, partly because the term variability bears different meanings and partly because of heterogeneity of studies, design, and methodological and reporting limitations.

All the following considerations separately and combined can benefit future research. The first is an RCT design to investigate the effect of strategies specifically targeting glucose variability reduction on mortality. However, it is hard to implement an intervention that could randomize patients to different variability cohorts, holding other glycemic outcomes equal. It might be acceptable to acknowledge that our understanding is likely to come from observational data. The second is a better understanding of what these different indicators measure and their relationship to the perceived quality of the BGL regulation process, the sampling frequency, and direction of change of BGLs. The third is a uniform indicator reference subset for glucose variability. The fourth is a better methodology and reporting practice, including adjustments and assumption checking pertaining to confounders and reporting association measures between severity of illness, measurement rates, and the variability indicators.

Open Access This article is distributed under the terms of the Creative Commons Attribution Noncommercial License which permits any noncommercial use, distribution, and reproduction in any medium, provided the original author(s) and source are credited. 


\section{References}

1. Van den Berghe G, Wilmer A, Hermans G, Meersseman W, Wouters PJ, Milants I, Van Wijngaerden E, Bobbaers H, Bouillon R (2006) Intensive insulin therapy in the medical ICU. N Engl J Med 354:449-461

2. Wiener RS, Wiener DC, Larson RJ (2008) Benefits and risks of tight glucose control in critically ill adults: a meta-analysis. JAMA 300:933-944

3. Griesdale DE, de Souza RJ, van Dam RM, Heyland DK, Cook DJ, Malhotra A, Dhaliwal R, Henderson WR, Chittock DR, Finfer S, Talmor D (2009) Intensive insulin therapy and mortality among critically ill patients: a metaanalysis including NICE-SUGAR study data. CMAJ 180:821-827

4. Finfer S, Chittock DR, Su SY, Blair D, Foster D, Dhingra V, Bellomo R, Cook D, Dodek P, Henderson WR, Hebert PC, Heritier S, Heyland DK, McArthur C, McDonald E, Mitchell I, Myburgh JA, Norton R, Potter J, Robinson BG, Ronco JJ (2009) Intensive versus conventional glucose control in critically ill patients. N Engl J Med 360:1283-1297

5. Marik PE, Preiser JC (2010) Toward understanding tight glycemic control in the ICU: a systematic review and metaanalysis. Chest 137:544-551

6. Eslami S, Abu-Hanna A, de Keizer NF, Bosman RJ, Spronk PE, de Jonge E, Schultz MJ (2010) Implementing glucose control in intensive care: a multicenter trial using statistical process control. Intensive Care Med 36:1556-1565

7. Preiser JC, Devos P, Ruiz-Santana S, Melot C, Annane D, Groeneveld J, Iapichino G, Leverve X, Nitenberg G, Singer P, Wernerman J, Joannidis M, Stecher A, Chiolero R (2009) A prospective randomised multi-centre controlled trial on tight glucose control by intensive insulin therapy in adult intensive care units: the Glucontrol study. Intensive Care Med 35:1738-1748

8. Eslami S, Abu-Hanna A, de Jonge E, de Keizer NF (2009) Tight glycemic control and computerized decisionsupport systems: a systematic review. Intensive Care Med 35:1505-1517

9. Pachler C, Plank J, Weinhandl H, Chassin LJ, Wilinska ME, Kulnik R, Kaufmann P, Smolle KH, Pilger E, Pieber TR, Ellmerer M, Hovorka R (2008) Tight glycaemic control by an automated algorithm with time-variant sampling in medical ICU patients. Intensive Care Med 34:1224-1230
10. Monnier L, Mas E, Ginet C, Michel F, Villon L, Cristol JP, Colette C (2006) Activation of oxidative stress by acute glucose fluctuations compared with sustained chronic hyperglycemia in patients with type 2 diabetes. JAMA 295:1681-1687

11. Brownlee M (2005) The pathobiology of diabetic complications: a unifying mechanism. Diabetes 54:1615-1625

12. Egi M, Bellomo R, Reade MC (2009) Is reducing variability of blood glucose the real but hidden target of intensive insulin therapy? Crit Care 13:302

13. Hermanides J, Vriesendorp TM, Bosman RJ, Zandstra DF, Hoekstra JB, Devries JH (2010) Glucose variability is associated with intensive care unit mortality. Crit Care Med 38:838-842

14. Vincent JL (2010) Blood glucose control in 2010: 110 to $150 \mathrm{mg} / \mathrm{dL}$ and minimal variability. Crit Care Med 38:993-995

15. Ali NA, O'Brien JM Jr, Dungan K, Phillips G, Marsh CB, Lemeshow S, Connors AF Jr, Preiser JC (2008) Glucose variability and mortality in patients with sepsis. Crit Care Med 36:2316-2321

16. Meyfroidt G, Keenan DM, Wang X, Wouters PJ, Veldhuis JD, Van den Berghe G (2010) Dynamic characteristics of blood glucose time series during the course of critical illness: effects of intensive insulin therapy and relative association with mortality. Crit Care Med 38:1021-1029

17. Harmsen RE, Spronk PE, Schultz MJ, Abu-Hanna A (2011) May frequency of blood glucose measurement be blurring the association between MAG and mortality? Crit Care Med 39:224

18. Chase JG, Shaw G, Le Compte A, Lonergan T, Willacy M, Wong XW, Lin J, Lotz T, Lee D, Hann C (2008) Implementation and evaluation of the SPRINT protocol for tight glycaemic control in critically ill patients: a clinical practice change. Crit Care 12:R49

19. Krinsley JS (2008) Glycemic variability: a strong independent predictor of mortality in critically ill patients. Crit Care Med 36:3008-3013

20. Lundelin K, Vigil L, Bua S, GomezMestre I, Honrubia T, Varela M (2010) Differences in complexity of glycemic profile in survivors and nonsurvivors in an intensive care unit: a pilot study. Crit Care Med 38:849-854
21. Eslami S, de Keizer NF, de Jonge E, Schultz MJ, Abu-Hanna A (2008) A systematic review on quality indicators for tight glycaemic control in critically ill patients: need for an unambiguous indicator reference subset. Crit Care 12:R139

22. Antonelli M, Azoulay E, Bonten M, Chastre J, Citerio G, Conti G, De Backer D, Lemaire F, Gerlach H, Hedenstierna G, Joannidis M, Macrae D, Mancebo J, Maggiore SM, Mebazaa A, Preiser JC, Pugin J, Wernerman J, Zhang H (2010) Year in review in Intensive Care Medicine 2009; I: Pneumonia and infections, sepsis, outcome, acute renal failure and acid base, nutrition and glycaemic control. Intensive Care Med 36:196-209

23. van den Berghe G, Wouters P, Weekers F, Verwaest C, Bruyninckx F, Schetz M, Vlasselaers D, Ferdinande P, Lauwers P, Bouillon R (2001) Intensive insulin therapy in the critically ill patients. N Engl J Med 345:1359-1367

24. Chase JG, Pretty CG, Pfeifer L, Shaw GM, Preiser JC, Le Compte AJ, Lin J, Hewett D, Moorhead KT, Desaive T (2010) Organ failure and tight glycemic control in the SPRINT study. Crit Care 14:R154

25. Jacka MJ, Torok-Both CJ, Bagshaw SM (2009) Blood glucose control among critically ill patients with brain injury. Can J Neurol Sci 36:436-442

26. Bagshaw SM, Bellomo R, Jacka MJ, Egi M, Hart GK, George C (2009) The impact of early hypoglycemia and blood glucose variability on outcome in critical illness. Crit Care 13:R91

27. Pidcoke HF, Wanek SM, Rohleder LS, Holcomb JB, Wolf SE, Wade CE (2009) Glucose variability is associated with high mortality after severe burn. J Trauma 67:990-995

28. Dossett LA, Cao H, Mowery NT, Dortch MJ, Morris JM Jr, May AK (2008) Blood glucose variability is associated with mortality in the surgical intensive care unit. Am Surg 74:679-685 (discussion 685)

29. Hirshberg E, Larsen G, Van Duker H (2008) Alterations in glucose homeostasis in the pediatric intensive care unit: hyperglycemia and glucose variability are associated with increased mortality and morbidity. Pediatr Crit Care Med 9:361-366 
30. Waeschle RM, Moerer O, Hilgers R, Herrmann P, Neumann P, Quintel M (2008) The impact of the severity of sepsis on the risk of hypoglycaemia and glycaemic variability. Crit Care 12:R129
31. Egi M, Bellomo R, Stachowski E, French CJ, Hart G (2006) Variability of blood glucose concentration and shortterm mortality in critically ill patients. Anesthesiology 105:244-252
32. Wintergerst KA, Buckingham B, Gandrud L, Wong BJ, Kache S, Wilson DM (2006) Association of

hypoglycemia, hyperglycemia, and glucose variability with morbidity and death in the pediatric intensive care unit. Pediatrics 118:173-179 